\title{
Classificação de cultivares de brássicas com relação à resistência à traça-das-crucíferas e à presença de glucosinolatos
}

\author{
Robson Thomaz Thuler ${ }^{(1)}$, Sergio Antonio De Bortoli( ${ }^{(1)}$ e Clara Beatriz Hoffmann-Campo ${ }^{(2)}$
}

\begin{abstract}
(1)Universidade Estadual Paulista, Dep. de Fitossanidade, CEP 14884-900 Jaboticabal, SP. E-mail: rthuler@hotmail.com, bortoli@fcav.unesp.br (2)Embrapa Soja, Caixa Postal 231, CEP 86001-970 Londrina, PR. E-mail: hoffmann@cnpso.embrapa.br
\end{abstract}

\begin{abstract}
Resumo - O objetivo deste trabalho foi avaliar o efeito de cultivares de repolho e de couve na biologia da traçadas-crucíferas; analisar a composição química das plantas, em relação aos glucosinolatos e aplicar a análise multivariada na classificação das cultivares. Foram utilizadas as cultivares: de repolho verde Chato-de-quintal e o híbrido Midori; de repolho roxo Roxo precoce e o híbrido Roxo TPC00682; e de couve-manteiga Geórgia (padrão de suscetibilidade) e Geórgia híbrido HS20. Foram avaliadas as características biológicas da praga: viabilidade e duração das fases larval e pupal, razão sexual, fecundidade das fêmeas, longevidade dos adultos e duração e viabilidade da fase de ovo, tendo-se calculado o potencial reprodutivo corrigido (PRC). Obtiveram-se as correlações entre os parâmetros pelo método de Pearson, e realizaram-se análises multivariadas de agrupamento e de componentes principais. Em cromatógrafo líquido, avaliaram-se a presença de sinigrina e de outros glucosinolatos nas plantas. A cultivar Geórgia e os híbridos HS20 e Roxo foram classificados como altamente suscetíveis; 'Roxo precoce' e 'Midori' como suscetíveis e, 'Chato-de-quintal' como moderadamente resistente à Plutella xylostella. As análises multivariadas proporcionam melhor classificação das cultivares, em razão do grau de resistência apresentado. Os materiais genéticos avaliados não apresentam a substância secundária sinigrina.
\end{abstract}

Termos para indexação: Plutella xylostella, repolho, couve, potencial reprodutivo, resistência de plantas.

\section{Brassicas cultivars classification in relation to resistance to diamondback moth and to the presence of glucosinolates}

\begin{abstract}
The objective of this work was to evaluate the effect of cabbage and kale cultivars on the diamondback moth biology; to analyze the plant chemical composition in relation to glucosinolates and to apply the multivariate analysis to classify cultivar resistance. Trials were conduct using: green cabbage cultivar Chato-de-quintal and hybrid Midori; purple cabbage Roxo precoce and purple hybrid TPC00682; kale cultivar Georgia (susceptibility standard) and hybrid HS20. The pest biological characteristics evaluated were: viability and the length of the larval and pupal period, sex ratio, female fertility, adult longevity and the length and viability of the egg phase; also it was calculated the corrected reproductive potential index (CRP). Pearson correlation was obtained, and the grouping and main components of multivariate analysis were applied. Sinigrin and other glucosinolates were evaluated by liquid chromatography. 'Georgia', 'Roxo' and HS20 hybrids were classified as highly susceptible; 'Roxo precoce' and Midori hybrid, as susceptible, and 'Chato-de-quintal' as moderately resistant to diamondback moth. Multivariate analyses provided a better way to classify cultivars in relation to different resistance degrees. Brassica genetic materials evaluated do not present sinigrin.
\end{abstract}

Index terms: Plutella xylostella, cabbage, kale, reproductive potential, plant resistance.

\section{Introdução}

A traça-das-crucíferas Plutella xylostella L. (Lepidoptera: Plutellidae) é o principal fator limitante do cultivo de crucíferas em áreas tropicais no mundo, em razão, principalmente, de seu ciclo curto e alto potencial reprodutivo, o que determina número anual elevado de gerações (Ulmer et al., 2002). O controle da traça-das-crucíferas é realizado com o uso de insetici- das, o que causa o aparecimento de populações resistentes (Castelo Branco et al., 2001). No Brasil, a resistência de P. xylostella a inseticidas foi relatada por Castelo Branco \& Gatehouse (1997), em relação aos diferentes princípios ativos comumente utilizados em seu controle. Recentemente, Castelo Branco \& Melo (2002) estudaram a resistência apresentada por populações de traça-das-crucíferas a abamectin, com base nos trabalhos de Zhang et al. (2001) e Iqbal et al. (1996), 
que haviam observado resistência dessa praga àquele inseticida, na China e Malásia, respectivamente.

Como alternativa ao uso de inseticidas, a utilização de cultivares resistentes tem assumido papel relevante no manejo da traça-das-crucíferas (Ulmer et al., 2002) e de outras pragas importantes para essa cultura (Picoaga et al., 2003).

A resistência de plantas à $P$. xylostella tem sido avaliada com base em duas características principais: a cerosidade da superfície foliar, determinada pelo teor de alcano, e o teor de sinigrina presente nas folhas (Eigenbrode et al., 1990, 1991; Spencer, 1996; Spencer et al., 1999; Ulmer et al., 2002). Entretanto, Eigenbrode et al. (1990) consideram que os mecanismos envolvidos nas características de resistência, relacionadas à quantidade de cera presente na superfície foliar, não são conclusivos.

A sinigrina presente em cultivares de brássicas, mesmo em baixos níveis, pode influenciar negativamente insetos polífagos como o pulgão Myzus persicae e a lagarta Mamestra configurata, mas pode não afetar algumas espécies, específicas de brássicas, como o pulgão Brevicoryne brassicae e a lagarta P. xylostella (Bodnaryk, 1997; Cole, 1997).

No Brasil, alguns resultados foram relatados quanto à resistência de diferentes cultivares e híbridos de repolho e couve à traça-das-crucíferas, no entanto, esses resultados são baseados principalmente em dados biológicos de desenvolvimento da praga (Barros, 1998; Barros \& Vendramim, 1999; Torres, 2004) e não em características da planta hospedeira.

Barros (1998) utilizou o índice potencial reprodutivo corrigido (PRC), para demonstrar a influência dos genótipos de brássicas na biologia de P. xylostella, tendo classificado as cultivares em grupos distintos, quanto ao grau de resistência apresentado. Torres (2004) também utilizou o índice PRC na classificação de cultivares de repolho, em relação à resistência contra a traça, e afirmou que esse é um parâmetro preciso para se avaliar a influência de genótipos na biologia da traça-das-crucíferas.

O objetivo deste trabalho foi avaliar o efeito de cultivares de repolho e couve na biologia da traçadas-crucíferas; analisar a composição química das plantas em relação à presença de glucosinolatos, e aplicar a análise multivariada na classificação das cultivares em relação à resistência a esse inseto.

\section{Material e Métodos}

O experimento foi conduzido no Laboratório de Biologia e Criação de Insetos (LBCI), da Faculdade de Ciências
Agrárias e Veterinárias, da Universidade Estadual Paulista (FCAV-Unesp), em Jaboticabal, entre junho e julho de 2004, a $25 \pm 1^{\circ} \mathrm{C}$, umidade relativa de $70 \pm 10 \%$ e fotófase de 14 horas.

As cultivares utilizadas na experimentação foram plantadas em área experimental com solo corrigido, segundo a necessidade de plantio, e foi realizada adubação de cobertura, com a fórmula 4-14-8 (NPK), aos 20 dias após o transplantio. As plantas foram constantemente irrigadas durante os períodos de estiagem, de acordo com o aspecto visual das folhas.

$\mathrm{Na}$ primeira fase do experimento foram utilizadas lagartas de primeiro ínstar de P. xylostella, oriundas da criação-estoque do laboratório, conforme Barros (1998), tendo-se utilizado folhas de couve-manteiga.

Foram utilizados: a cultivar de repolho (Brassica oleracea capitata) verde Chato-de-quintal (Top Seed - Agristar) (CQ) e o híbrido Midori (Tokita - Agristar); a cultivar de repolho roxo Roxo precoce (Top Seed Agristar) e o híbrido Roxo TPC00682 (Top Seed Agristar); e as cultivares de couve-manteiga (Brassica oleracea acephala) Geórgia (Top Seed - Agristar) e Geórgia híbrido HS20 (Horticeres); 'Geórgia' foi utilizada como padrão de suscetibilidade. Insetos retirados da criação foram mantidos nessas cultivares durante uma geração e, posteriormente, procedeu-se à experimentação.

Para cada cultivar, foram utilizadas 20 placas de Petri ( $9 \mathrm{~cm}$ de diâmetro), com 20 repetições com um disco de folha de $8 \mathrm{~cm}$ de diâmetro, sobre papel filtro levemente umedecido com água. Sobre os discos foliares, foram colocadas 12 lagartas de primeiro ínstar de P. xylostella, recém-emergidas e, posteriormente, as placas foram fechadas com filme de plástico, para manter a umidade e evitar a fuga dos insetos. As avaliações da viabilidade e duração larval iniciaram-se a partir do terceiro dia, em razão do hábito minador do inseto no primeiro ínstar. As lagartas foram mantidas nas placas até atingirem a fase de pupa. Em seguida, os insetos foram transferidos para placas de plástico com orifício (Elisa) e foram observados até a emergência dos adultos, tendo-se acompanhado a duração, a viabilidade, o peso das pupas com 24 horas de formadas e a razão sexual dos insetos que emergiram.

Adultos emergidos do experimento anterior foram sexados e transferidos para gaiolas de postura, com cinco repetições por cultivar, tendo-se colocado dois casais por gaiola. Nessa fase, foi observada a fecundidade das fêmeas (número de ovos por fêmea), durante cinco dias (período de postura viável) e a longevidade dos adultos. Em seguida, os ovos obtidos foram colocados em pla- 
cas de Petri e observados até a eclosão, determinou-se, então, a viabilidade.

Os dados obtidos foram analisados, os valores foram submetidos, inicialmente, à análise de variância, e as médias foram comparadas pelo teste de Tukey, a 5\% de probabilidade. Para verificar a possível influência dos tratamentos, no desenvolvimento e aumento da população, foi calculado o índice potencial reprodutivo corrigido (PRC), segundo Barros (1998): $\mathrm{PRC}=(\mathrm{rs} \times \mathrm{A})^{\mathrm{n}}$, em que: rs é a razão sexual $=\mathrm{n}^{\mathrm{o}}$ de fêmeas $/ \mathrm{n}^{\circ}$ de adultos; A é o número de adultos aptos à reprodução, determinado para cada cultivar, em função do número médio de ovos por fêmea (no período determinado), corrigido pelas viabilidades das fases de ovo, larva e pupa; n é o número de gerações do inseto, em 30 dias, obtido pelo quociente: 30 (dias do mês)/duração total da fase imatura do inseto (dias de duração de ovo + larva + pupa).

Para verificação dos parâmetros que mais influenciaram a resistência das cultivares à $P$. xylostella, foram obtidas as correlações entre os parâmetros em função de todas as cultivares, pelo método de Pearson, com o programa Sigmastat versão 3.1 (Systat Softwere Inc., 2004). Realizou-se, também, a análise de agrupamento (AA) (Sneath \& Sokal, 1973) e a análise de componentes principais (ACP) (Jackson, 1991), para classificar as cultivares que apresentassem máxima similaridade dentro dos grupos e máxima dissimilaridade entre os grupos, com uso do programa Statistica versão 6.0 (Statsoft Inc., 2004).

Para a análise da presença de glucosinolatos, especialmente a sinigrina, substância reconhecidamente envolvida no processo de estímulo alimentar de preferência de P. xylostella (Thorsteinson, 1958), foram coletadas $60 \mathrm{~g}$ de amostras frescas (folha) de cada cultivar, dos quais $30 \mathrm{~g}$ foram congeladas e $30 \mathrm{~g}$ liofilizadas. As amostras congeladas foram mantidas em congelador $\left(-20^{\circ} \mathrm{C}\right)$ como reserva, e as liofilizadas, em tubos Eppendorf de $2 \mathrm{~mL}$, foram levadas à Embrapa Soja para processamento e análise.

Aproximadamente $4 \mathrm{~g}$ de amostra liofilizada de cada cultivar foram pesados, tendo-se adicionado $100 \mathrm{~mL}$ de
$\mathrm{EtOH} 70 \%$ a $50^{\circ} \mathrm{C}$. O material foi resfriado, homogeneizado, aquecido por $2,5 \mathrm{~min}$, resfriado e filtrado. O resíduo foi lavado duas vezes com $25 \mathrm{~mL} \mathrm{EtOH}$ $70 \%$, e o filtrado foi concentrado em rotavapor até aproximadamente $25 \mathrm{~mL}$.

Foi utilizado o protocolo de Betz \& Fox (1994), pelo qual cada amostra concentrada foi passada numa coluna aberta preenchida pela resina adsorbante $\mathrm{C} 18$, que foi lavada com $25 \mathrm{~mL} \mathrm{MeOH} 100 \%$ e condicionada com $25 \mathrm{~mL}$ de IPC-A 0,01M (tetrabutylammonium sulfate). Em seguida, adicionaram-se $25 \mathrm{~mL}$ da amostra concentrada à coluna, $20 \mathrm{~mL}$ de $\mathrm{H}_{2} \mathrm{O}$ para lavar a coluna e $30 \mathrm{~mL}$ MeOH:H20 (55:45) para eluição dos glucosinolatos.

Cada amostra foi analisada em cromatógrafo líquido de alta eficiência (CLAE) (Shimadzu), com coluna de fase reversa CLS-ODS-C18 de diâmetro interno de 4,6x250 mm de comprimento. Na eluição foi utilizado um gradiente linear, em que a fase móvel 'A' foi composta de $12 \%$ de $\mathrm{MeOH}$ por $88 \%$ IPC-A $0,005 \mathrm{M}$ e a fase móvel 'B' de $34 \% \mathrm{MeOH}$ por $66 \%$ IPC-A $0,005 \mathrm{M}$. O sistema inicial do gradiente foi constituído de $100 \%$ de fase móvel 'A', atingiu, aos 20 min, o valor de 100\% de fase móvel 'B', e permaneceu assim até os $35 \mathrm{~min}$. Aos 40 min, voltou à condição inicial de $100 \%$ de fase. O fluxo do solvente foi de $1 \mathrm{~mL}$ por minuto, e a absorção UV foi medida a $237 \mathrm{~nm}$. Como padrão, utilizou-se uma solução de $1 \mathrm{mg}$ de Sinigrina (Sigma) dissolvida em $1 \mathrm{~mL}$ de $\mathrm{MeOH}$, e uma amostra de $20 \mu \mathrm{L}$ foi injetada no CLAE e analisada nas mesmas condições das amostras de tecido vegetal.

\section{Resultados e Discussão}

Foram observadas diferenças significativas na duração e viabilidade larval e pupal e peso de pupa. Razão sexual, fecundidade e longevidade não sofreram alteração em razão das cultivares (Tabelas 1 e 2).

Os insetos alimentados com repolho 'Chato-dequintal' apresentaram o maior período larval (8,2 dias) e diferiram, significativamente, dos demais tratamen-

Tabela 1. Duração e viabilidade $( \pm \mathrm{IC})$ dos períodos larval e pupal de Plutella xylostella, alimentada com folhas de diferentes cultivares de couve e repolho ${ }^{(1)}$.

\begin{tabular}{|c|c|c|c|c|c|c|c|c|}
\hline \multirow[t]{2}{*}{ Genótipo } & \multicolumn{4}{|c|}{ Duração (dias) } & \multicolumn{4}{|c|}{ Viabilidade (\%) } \\
\hline & $\mathrm{n}$ & Larva & $\mathrm{n}$ & Pupa & $\mathrm{n}$ & Larva & $\mathrm{n}$ & Pupa \\
\hline Chato-de-quintal & 147 & $8,2 \pm 0,37 a$ & 114 & $4,2 \pm 0,16 \mathrm{a}$ & 240 & $61,3 \pm 9,86 b$ & 147 & $70,7 \pm 14,50 b$ \\
\hline Midori & 199 & $7,2 \pm 0,28 b$ & 180 & $4,0 \pm 0,08 \mathrm{ab}$ & 240 & $82,8 \pm 7,25 a$ & 199 & $91,1 \pm 10,72 \mathrm{a}$ \\
\hline Roxo precoce & 201 & $7,2 \pm 0,13 b$ & 179 & $3,8 \pm 0,12 b$ & 240 & $83,7 \pm 7,72 \mathrm{a}$ & 201 & $88,6 \pm 5,55 \mathrm{a}$ \\
\hline HS20 & 211 & $6,8 \pm 0,11 \mathrm{~b}$ & 191 & $3,4 \pm 0,10 \mathrm{c}$ & 240 & $88,0 \pm 5,09 a$ & 211 & $90,8 \pm 4,17 \mathrm{a}$ \\
\hline Geórgia & 214 & $6,8 \pm 0,13 b$ & 203 & $3,3 \pm 0,09 \mathrm{c}$ & 240 & $89,3 \pm 6,17 \mathrm{a}$ & 214 & $94,6 \pm 4,24 a$ \\
\hline Híbrido Roxo & 212 & $6,9 \pm 0,09 b$ & 182 & $3,4 \pm 0,13 \mathrm{c}$ & 240 & $88,3 \pm 6,09 \mathrm{a}$ & 212 & $86,1 \pm 6,72 \mathrm{ab}$ \\
\hline
\end{tabular}

${ }^{(1)}$ Médias seguidas por letras iguais, na coluna, não diferem entre si pelo teste de Tukey, a 5\% de probabilidade. 
tos (Tabela 1). Essa cultivar também foi a que ocasionou o período pupal mais longo, com 'Midori' (Md), seguida de repolho 'Roxo precoce' (Rx) (Tabela 1).

Além da duração dos períodos larval e pupal mais elevados, em repolho 'Chato-de-quintal', observouse também a menor viabilidade nessas fases. As larvas alimentadas com essa cultivar apresentaram $61,3 \%$ de viabilidade, e as pupas formadas nesse mesmo tratamento resultaram em 70,7\% de adultos, valores que diferiram significativamente das médias das demais cultivares, exceto do híbrido Roxo (Tabela 1).

As respostas observadas para os parâmetros citados podem estar relacionadas a compostos químicos ativos, metabolizados pela planta, que se tornam toxinas fisiológicas e causam a clássica antibiose, ou substâncias deterrentes que evitam a alimentação do inseto reduzindo sua viabilidade, como sugerido por Eigenbrode et al. (1990).

As pupas formadas nos tratamentos com 'Geórgia' apresentaram o maior peso médio (Tabela 2). O peso de pupa está diretamente relacionado às outras características estudadas, uma vez que foram observadas altas correlações entre esse índice e a duração larval, viabilidade larval, viabilidade pupal, razão sexual e fecundidade (Tabela 3). Biologicamente, há coerência nos resultados apresentados, pois 'Geórgia' é utilizada na criação massal de $P$. xylostella, portanto é nutricionalmente mais adequada.

A análise de correlação de Pearson (Tabela 3) mostra a dependência entre as características biológicas avaliadas e indica a tendência no desenvolvimento do inseto e a relevância dessas características para avaliação da influência do alimento disponibilizado no desenvolvimento do inseto.

As altas correlações negativas, observadas entre o índice PRC e as durações larval e pupal (Tabela 3), demonstram que essas características foram as que mais contribuíram para a elevação dos valores do índice para cada cultivar, isto porque durações menores implicam em maior número de gerações e em menor tempo e influenciam diretamente a potência n, utilizada para o cálculo do PRC.

Cole (1997) correlacionou o substrato alimentar à razão intrínseca de crescimento da população de um pulgão, específico de brássicas Brevicoryne brassicae, e um polífago Myzus persicae, e observou que o crescimento populacional da primeira espécie aumentou com as quantidades crescentes de glucosinolatos nas cultivares, enquanto para a segunda espécie não houve alteração em conseqüência desses compostos, o que indica,

Tabela 2. Peso médio de pupa (g), razão sexual, fecundidade e longevidade de adultos (dias) ( \pm IC) de Plutella xylostella, alimentada com folhas de diferentes cultivares de couve e repolho ${ }^{(1)}$.

\begin{tabular}{|c|c|c|c|c|c|c|c|c|}
\hline \multirow[t]{2}{*}{ Genótipo } & \multicolumn{2}{|c|}{ Peso } & \multicolumn{2}{|c|}{ Razão sexual } & \multicolumn{2}{|c|}{ Fecundidade } & \multicolumn{2}{|c|}{ Longevidade } \\
\hline & $\mathrm{n}$ & Pupa & $\mathrm{n}$ & Adulto & $\mathrm{n}$ & Adulto & $\mathrm{n}$ & Adulto \\
\hline Chato-de-quintal & 147 & $0,0049 \mathrm{~d}$ & 114 & $0,3 \pm 0,12 \mathrm{a}$ & 10 & $109,5 \pm 39,71 a$ & 20 & $7,1 \pm 0,91 \mathrm{a}$ \\
\hline Midori & 199 & $0,0054 \mathrm{ab}$ & 180 & $0,4 \pm 0,08 \mathrm{a}$ & 10 & $145,8 \pm 13,47 a$ & 20 & $7,7 \pm 1,22 \mathrm{a}$ \\
\hline Roxo precoce & 201 & $0,0051 \mathrm{~cd}$ & 179 & $0,5 \pm 0,09 \mathrm{a}$ & 10 & $149,3 \pm 8,93 \mathrm{a}$ & 20 & $7,3 \pm 0,20 \mathrm{a}$ \\
\hline $\mathrm{HS} 20$ & 211 & $0,0053 \mathrm{bc}$ & 191 & $0,5 \pm 0,08 \mathrm{a}$ & 10 & $150,5 \pm 22,41 \mathrm{a}$ & 20 & $6,8 \pm 0,57 \mathrm{a}$ \\
\hline Geórgia & 214 & $0,0055 \mathrm{a}$ & 203 & $0,5 \pm 0,08 \mathrm{a}$ & 10 & $147,8 \pm 14,45 \mathrm{a}$ & 20 & $7,4 \pm 0,25 \mathrm{a}$ \\
\hline Híbrido Roxo & 212 & $0,0051 \mathrm{~cd}$ & 182 & $0,4 \pm 0,07 \mathrm{a}$ & 10 & $132,2 \pm 26,16 a$ & 20 & $6,6 \pm 1,21 \mathrm{a}$ \\
\hline
\end{tabular}

${ }^{(1)}$ Médias seguidas por letras iguais, na coluna, não diferem entre si pelo teste de Tukey, a 5\% de probabilidade.

Tabela 3. Coeficientes de correlação (r) e respectivas probabilidades (p) entre características biológicas de Plutella xylostella, alimentada com diferentes cultivares de couve e repolho.

\begin{tabular}{|c|c|c|c|c|c|c|c|c|}
\hline Variável & & $\begin{array}{c}\text { Duração } \\
\text { larval }\end{array}$ & $\begin{array}{c}\text { Viabilidade } \\
\text { larval }\end{array}$ & $\begin{array}{c}\text { Duração } \\
\text { pupal }\end{array}$ & $\begin{array}{c}\text { Viabilidade } \\
\text { pupal }\end{array}$ & $\begin{array}{l}\text { Razão } \\
\text { sexual }\end{array}$ & Fecundidade & $\mathrm{PRC}^{(1)}$ \\
\hline \multirow[t]{2}{*}{ Peso de pupa } & $\mathrm{r}$ & $-0,84$ & 0,97 & - & 0,96 & 0,93 & 0,92 & - \\
\hline & $\mathrm{p}$ & 0,036 & 0,001 & $*$ & 0,002 & 0,006 & 0,009 & $*$ \\
\hline \multirow[t]{2}{*}{ Duração larval } & $\mathrm{r}$ & - & $-0,94$ & 0,96 & $-0,82$ & $-0,86$ & - & $-0,86$ \\
\hline & $\mathrm{p}$ & - & 0,005 & 0,002 & 0,04 & 0,03 & $*$ & 0,026 \\
\hline \multirow[t]{2}{*}{ Viabilidade larval } & $\mathrm{r}$ & - & - & $-0,83$ & 0,93 & 0,93 & 0,85 & - \\
\hline & $\mathrm{p}$ & - & - & 0,042 & 0,007 & 0,007 & 0,030 & $*$ \\
\hline \multirow[t]{2}{*}{ Duração pupal } & $\mathrm{r}$ & - & - & - & - & - & - & $-0,85$ \\
\hline & $\mathrm{p}$ & - & - & - & $*$ & $*$ & $*$ & 0,032 \\
\hline \multirow[t]{2}{*}{ Viabilidade pupal } & $\mathrm{r}$ & - & - & - & - & 0,91 & 0,95 & - \\
\hline & $\mathrm{p}$ & - & - & - & - & 0,011 & 0,004 & $*$ \\
\hline \multirow{2}{*}{ Razão sexual } & $r$ & - & - & - & - & - & 0,95 & - \\
\hline & $\mathrm{p}$ & - & - & - & - & - & 0,004 & $*$ \\
\hline
\end{tabular}

(1)Potencial reprodutivo corrigido. *Significativo a $5 \%$ de probabilidade. 
como nos resultados obtidos neste trabalho, que o substrato alimentar está intimamente ligado às respostas de resistência de plantas a insetos.

Por meio do PRC, verificou-se uma tendência de separação entre 'Chato-de-quintal' (Figura 1 A) e as demais cultivares (Figura 1 B), no entanto, essa divisão foi dificultada pela proximidade entre os dados, bem como pelos grandes intervalos de confiança (IC) apresentados para cada cultivar, que tornam a tendência menos relevante e impossibilitam a classificação.

Pela análise de agrupamento, observou-se que 'Chato-de-quintal' apresenta maior contraste em relação às demais cultivares e não pertence a nenhum grupo. 'Geórgia' e o híbrido HS20 de couve foram similares e próximas ao híbrido Roxo de repolho. Outro grupo foi formado pela 'Roxo precoce' e repolho verde 'Midori' (Figura 2). Com base nesses resultados, sugere-se a divisão das cultivares estudadas em três grupos distintos, na seguinte classificação: repolho verde 'Chato-de-quintal' como moderadamente resistente (MR); repolho 'Roxo precoce' e híbrido Midori como suscetíveis (S); e couve-manteiga 'Geórgia', 'Geórgia' híbrido HS20 e híbrido Roxo como altamente suscetíveis (AS).

Por meio da análise dos componentes principais (ACP), foi possível visualizar melhor o contraste da couve 'Geórgia' e 'Geógia' híbrido HS20 com o repolho verde

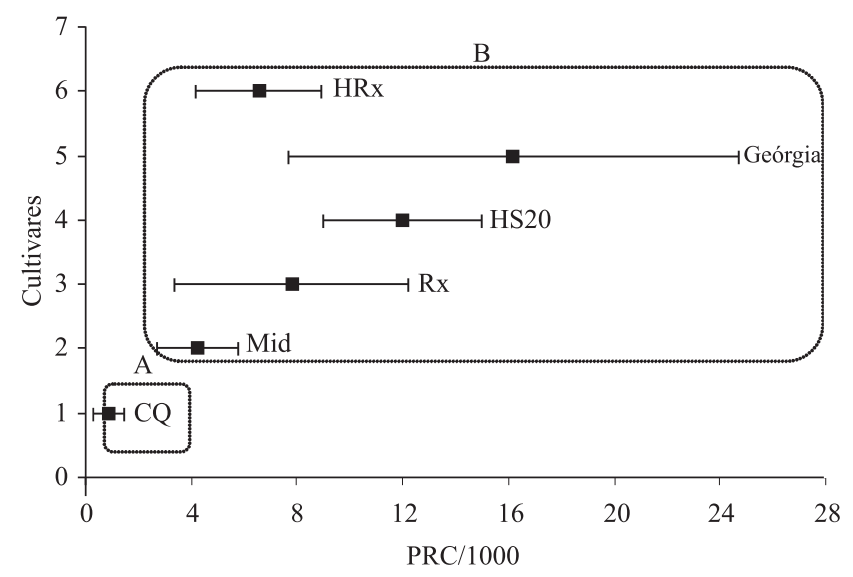

Figura 1. Potencial reprodutivo corrigido (PRC) de Plutella xylostella, alimentada com diferentes cultivares de repolho e couve. CQ: Chato-de-quintal; Mid: híbrido Midori; Rx: Roxo precoce; HS20: híbrido HS20; HRx: híbrido Roxo; valores de PRC transformados por PRC/1.000, em razão dos altos valores apresentados pelo índice. $\mathrm{p}=0,05$.
'Chato-de-quintal'. Verifica-se, também, o forte contraste entre 'Chato-de-quintal', 'Geórgia' e 'HS20' (Figura 3).

O repolho 'Chato-de-quintal' apresentou as maiores correlações com as características duração larval $(-0,96)$ e pupal (-0,83); 'Geórgia' e 'HS20' estão correlacionadas com a viabilidade larval $(0,95)$ e pupal $(0,96)$, peso pupal $(0,82)$, razão sexual $(0,91)$ e fecundidade $(0,91)$; o eixo CP1 é responsável pela retenção de $72,8 \%$ da variabilidade contida nas variáveis originais (Figura 3).

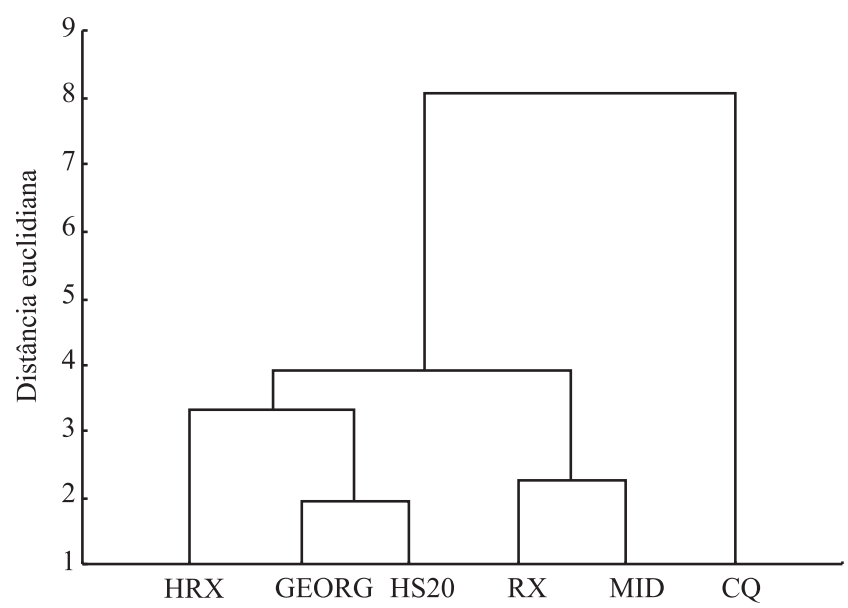

Figura 2. Dendrograma dos grupos resultantes da análise multivariada de agrupamento. CQ: Chato-de-quintal; Mid: híbrido Midori; RX: Roxo precoce; HS20: híbrido HS20; Georg: Geórgia; HRX: híbrido Roxo.

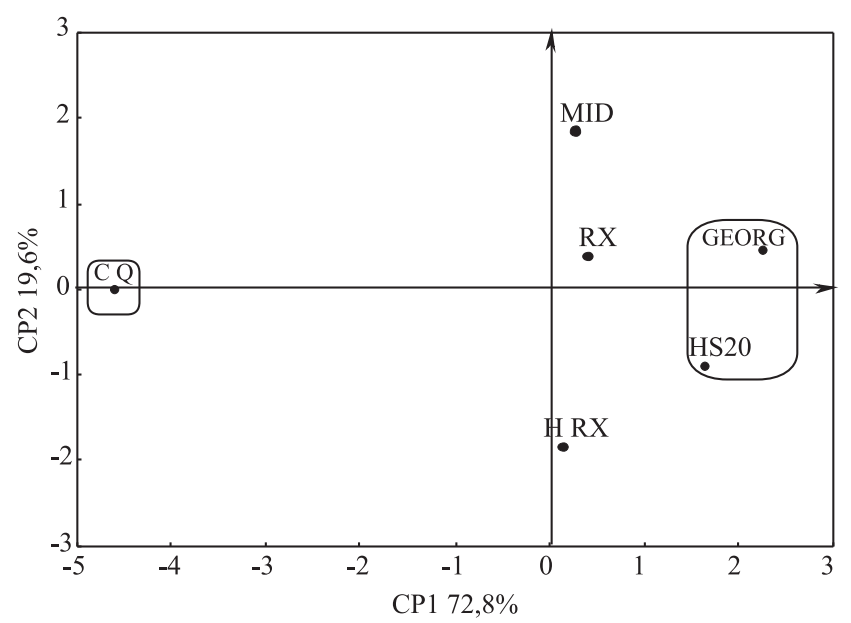

Figura 3. Distribuição das cultivares segundo a análise das componentes principais. CQ: Chato-de-quintal; Mid: híbrido Midori; RX: Roxo precoce; HS20: híbrido HS20; Georg: Geórgia; HRX: híbrido Roxo. 
Pode-se inferir, ainda, que a viabilidade larval e pupal, peso de pupa, razão sexual e fecundidade são maiores para os insetos alimentados com 'Geórgia' e 'HS20', enquanto as durações larval e pupal apresentam os menores valores, nessas mesmas cultivares. O inverso desta afirmação é observado para 'Chato-de-quintal'.

Em relação à $\mathrm{CP} 2$, apenas a longevidade apresentou alta correlação $(-0,98)$, e este componente foi responsável pela retenção de $19,6 \%$ da variabilidade total existente nas variáveis originais (Figura 3).

Barros (1998) e Torres (2004), com base apenas no PRC, obtiveram respostas diferentes para 'Chato-de-quintal', classificada em seus trabalhos como suscetível. 'Midori' também foi classificada de maneira diferente por Barros (1998). Em sua classificação, com base principalmente no PRC, a cultivar encontra-se no grupo das MR, no entanto, apesar de nas análises de PRC aproximar-se bastante do repolho verde 'Chato-de-quintal', classificado neste trabalho como MR, na análise de grupamento 'Midori' mostrou maior similaridade com o grupo das susctíveis (S). Além disso, na análise de componentes principais, não foi possível distingui-la num grupo discrepante. Em comparação com testes feitos também por Barros (1998), 'Roxo precoce' recebeu a mesma classificação $\mathrm{S}$, obtida neste trabalho.

Em relação às diferenças encontradas entre os resultados dos trabalhos de Barros (1998), Torres (2004), e os obtidos neste trabalho, há de se considerar que a característica de resistência é definida, conceitualmente, como relativa, portanto mutável, em razão do grupo teste. Adicionalmente, as condições abióticas como ambiente e adubação, que variam entre as regiões onde foram realizados os testes, podem influenciar nos resultados (Lara, 1991).

Quanto à análise das amostras das cultivares em CLAE, pelo cromatograma e pelo espectro obtido para sinigrina (Figura 4), pode-se afirmar que não foram observados traços de sinigrina em nenhuma das amostras analisadas. Porém, algumas substâncias com espectros de glucosinolatos foram observadas em algumas amostras. Comparando-se os espectros com os obtidos por Kiddle et al. (2001), observou-se serem estes semelhantes aos de neo-glucobracissin (tempo de retenção $=8,18$ min relativos), glucobracissin (tempo de retenção entre 12,79 e 14,69 min relativos) e 4-metoxy 3-indol metil glucosinolato (tempo de retenção entre 23,93 e 25,75 min relativos) (Tabela 4). Em geral, os traços com espectro de glucosinolato foram pequenos, porém o repolho híbrido Roxo apresentou traço de uma substância com espectro semelhante ao do glucobracissin, comparativamente mais elevado.

Apesar de terem sido encontrados vários glucosinolatos com espectros semelhantes aos observados por Kiddle et al. (2001), suas funções relativas à resistência de plantas à traça-das-crucíferas não são esclarecidas neste trabalho, bem como em outros, que

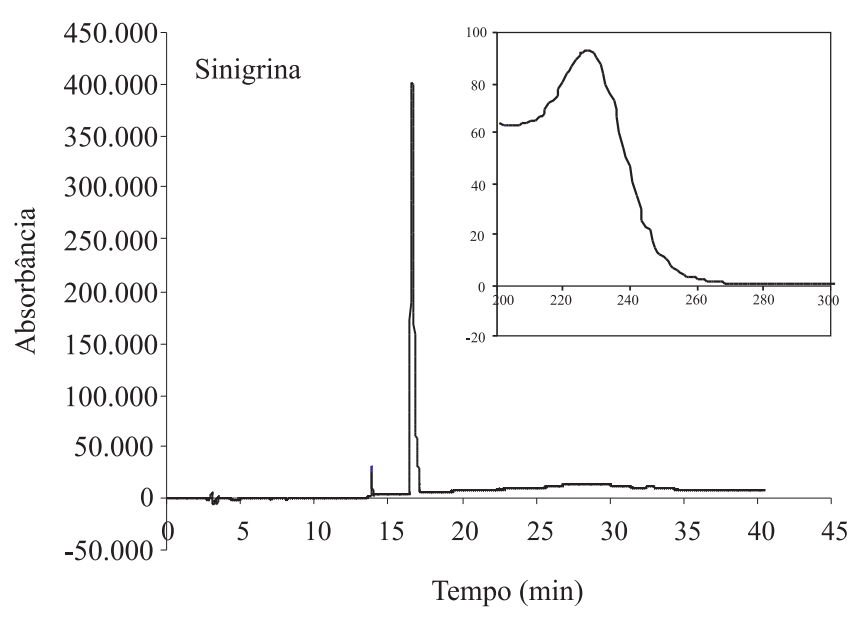

Figura 4. Cromatograma e espectro da amostra pura de sinigrina.

Tabela 4. Glucosinolatos encontrados em diferentes cultivares de couve e repolho.

\begin{tabular}{lccc}
\hline Genótipo & & Tempo de retenção (minutos relativos) \\
\cline { 2 - 4 } & (neo-glucobracissin) & 12,79-14,69 & 23,93-25,75 \\
(glucobracissin) & 4-metoxy 3-indol metil glucosinalato & + \\
\hline Chato-de-quintal & - & + & + \\
Midori & - & + & + \\
Roxo precoce & + & + & + \\
HS20 & - & + & + \\
Geórgia & - & + & - \\
Híbrido Roxo & - & & + \\
\hline
\end{tabular}


se referem à presença de diferentes glucosinolatos em várias espécies de brássicas (Elfakir \& Dreux, 1996; Tolrà et al., 2000; Troyer et al., 2001).

A constatação de ausência de sinigrina nas seis cultivares (Tabela 4) é de grande relevância, pois segundo Thorsteinson (1958), sua função primordial nas plantas era de defesa contra herbívoros, e com a co-evolução, insetos específicos de crucíferas, como P. xylostella, passaram a utilizá-la como fonte de atratividade e estímulo alimentar.

Spencer (1996) também comprovou a ação atrativa de sinigrina, para P. xylostella, quando observou que esta substância, aliada a compostos cerosos (alcanos), aumenta significativamente a preferência para oviposição dessa praga.

Pelos resultados, observa-se que a ausência de sinigrina nas plantas não alterou as respostas, em relação à resistência das cultivares e híbridos estudados, à traça-das-crucíferas. Tais resultados estão de acordo com Bodnaryk (1997), que em cultivares de brássicas com baixo teor dos glucosinolatos sinigrina e sinalbina, não observaram alterações na suscetibilidade à Phyllotreta cruciferae Goeze e P. xylostella (específicos de crucíferas), mas observaram acréscimo de cinco vezes na suscetibilidade dessas cultivares à Mamestra configurata Wlk. (inseto polífago).

A ausência de sinigrina nas seis cultivares analisadas pode ser também resultado dos excessivos melhoramentos realizados, para aumentar a produtividade, como observado para espécies de brássicas produtoras de óleo, para as quais os programas de melhoramento visavam reduzir os teores de glucosinolatos e obter maior produtividade (Bodnaryk, 1997).

\section{Conclusões}

1. A cultivar de repolho verde Chato-de-quintal é classificada como moderadamente resistente à Plutella xylostella.

2. As análises de agrupamento e de componentes principais proporcionam melhor classificação das cultivares do que o índice potencial reprodutivo corrigido.

3. Nenhuma das cultivares apresenta a substância secundária sinigrina, apesar de apresentarem outros glucosinolatos, cujas funções na biologia dos insetos ainda são desconhecidas.

\section{Agradecimentos}

À Fapesp, pela concessão da bolsa de estudos, reserva técnica e auxílio-pesquisa para o desenvolvimento deste trabalho; ao Prof. Dr. Antônio Sérgio Ferraudo, pela aplicação das análises multivariadas.

\section{Referências}

BARROS, R. Efeito de cultivares de repolho Brassica oleracea var. capitata (L.) na biologia da traça-das-crucíferas, Plutella xylostella (L., 1758) e do parasitóide Trichogramma pretiosum Riley, 1879. 1998. 98p. Tese (Doutorado) - Universidade de São Paulo, Piracicaba.

BARROS, R.; VENDRAMIM, J.D. Efeito de cultivares de repolho, utilizadas para criação de Plutella xylostella (L.) (Lepidoptera: Plutellidae), no desenvolvimento de Trichogramma pretiosum Riley (Hymenoptera: Trichogrammatidae). Anais da Sociedade Entomológica do Brasil, v.28, p.469-476, 1999.

BETZ, J.M.; FOX, W.D. High-performance liquid chromatographic determination of glucosinolates in Brassica vegetables. In: HUANG, M.-T.; OSAWA, T.; HO, C.T.; ROSEN, R.T. (Ed.). Food phytochemicals for cancer prevention $I$ : fruits and vegetables. Washington: Oxford University Press, 1994. p.181-195.

BODNARYK, R.P. Will low glucosinolate cultivars of the mustards Brassica juncea and Sinapis alba be vulnerable to insect pests? Canadian Journal of Plant Science, v.77, p.283-287, 1997.

CASTELO BRANCO, M.; FRANÇA, F.H.; MEDEIROS, M.A.; LEAL, J.G.T. Uso de inseticidas para o controle da traça-do-tomateiro e traça-das-crucíferas: um estudo de caso. Horticultura Brasileira, v.19, p.60-63, 2001.

CASTElo BRANCO, M.; GATEHOUSE, A.G. Insecticide resistance in Plutella xylostella (Lepidoptera: Yponomeutidae) in the Federal District, Brazil. Anais da Sociedade Entomológica do Brasil, v.26, p.75-79, 1997.

CASTELO BRANCO, M.; MELO, C.A. de. Resistência a abamectin e cartap em populações de traça-das-crucíferas. Horticultura Brasileira, v.20, p.541-543, 2002.

COLE, R.A. The relative importance of glucosinolates and amino acids to the development of two aphid pests Brevicoryne brassicae and Myzus persicae on wild and cultivated brassica species. Entomologia Experimentalis et Applicata, v.85, p.121-133, 1997.

EIGENBRODE, S.D.; SHELTON, A.M.; DICKSON, M.H. Two types of resistance to the diamondback moth (Lepidoptera: Plutellidae) in cabbage. Environmental Entomology, v.19, p.10861090, 1990.

EIGENBRODE, S.D.; STONER, K.A.; SHELTON, A.M.; KAIN, W.C. Characteristics of glossy leaf waxes associated with resistance to diamondback moth (Lepidoptera: Plutellidae) in Brassica oleracea. Journal of Economic Entomology, v.84, p.1609-1618, 1991.

ELFAKIR, C.; DREUX, M. Simultaneous analysis of intact and desulfated glucosinolates with a porous graphitized carbon column. Journal of Chromatography A, v.727, p.71-82, 1996. 
IQBAL, M.; VERKERK, R.H.J.; FURLONG, M.J.; ONG, P.C.; RAHMAN, S.A.; WRIGHT, D.J. Evidence for resistance to Bacillus thuringiensis (Bt) subsp. kurstaki HD-1, Bt subsp. aizawai and abamectin in field populations of Plutella xylostella from Malaysia. Pesticide Science, v.48, p.89-97, 1996.

JACKSON, J.E. A user's guide to principal components. New York: Wiley, 1991. 569p.

KIDDLE, G.; BENNETT, R.N.; BOTTING, N.P.; DAVIDSON, N.E.; ROBERTSON, A.A.B.; WALLSGROVE, R.M. Highperformance liquid chromatographic separation of natural and synthetic desulphoglucosinolates and their chemical validation by UV, NMR and chemical ionisation-MS methods. Phytochemical Analysis, v.12, p.226-242, 2001.

LARA, F.M. Princípios de resistência de plantas a insetos. São Paulo: Ícone, 1991. 336p.

PICOAGA, A.; CARTEA, M.E.; SOENGAS, P.; MONETTI, L.; ORDÁS, A. Resistance of kale populations to lepidopterous pests in Northwestern Spain. Journal of Economic Entomology, v.96, p.143-147, 2003.

SNEATH, P.H.A.; SOKAL, R.R. Numerical taxonomy. San Francisco: W. H. Freeman, 1973. 573p.

SPENCER, J.L. Waxes enhance Plutella xylostella oviposition in response to sinigrin and cabbage homogenates. Entomologia Experimentalis et Applicata, v.81, p.165-173, 1996.

SPENCER, J.L.; PILLAI, S.; BERNAYS, E.A. Synergism in the oviposition behavior of Plutella xylostella: sinigrin and wax compounds. Journal of Insect Behavior, v.12, p.483-500, 1999.

STATSOFT. Statistica: data analysis software system, version 7. Tulsa, 2004. Disponível em: <http://www.statsoft.com>. Acesso em: 25 jul. 2005.
SYSTAT SOFTWARE. SigmaStat: advisory statistics for scientists, version 1. San Jose, 2004. Disponível em: <http://www.systat.com/ products/SigmaStat/>. Acesso em: 10 set. 2004.

THORSTEINSON, A.J. The chemotactic influence of plant constituents on feeding by phytophagous insects. Entomologia Experimentalis et Applicata, v.1, p.23-27, 1958.

TOLRÀ, R.P.; ALONSO, R.; POSCHENRIEDER, C.; BARCELÓ, D.; BARCELÓ, J. Determination of glucosinolates in rapeseed and Thlaspi caerulescens plants by liquid chromatography-atmospheric pressure chemical ionization mass spectrometry. Journal of Chromatography A, v.889, p.75-81, 2000.

TORRES, A.L. Efeito de cultivares de repolho e extratos aquosos vegetais na biologia de Plutella xylostella (L.) e no parasitóide Oomyzus sokolowskii (Kurdjumov). 2004. 109p. Tese (Doutorado) - Universidade Estadual Paulista, Jaboticabal.

TROYER, J.K.; STEPHENSON, K.K.; FAHEY, J.W. Analysis of glucosinolates from broccoli and other cruciferous vegetables by hydrophilic interaction liquid chromatography. Journal of Chromatography A, v.919, p.299-304, 2001.

ULMER, B.; GILLOTT, C.; WOODS, D.; ERLANDSON, M. Diamondback moth, Plutella xylostella (L.), feeding and oviposition preferences on glossy and waxy Brassica rapa (L.) lines. Crop Protection, v.21, p.327-331, 2002.

ZHANG, X.Y.; JIE, H.; YE, C.Y.; XUE, Y. Monitoring on the resistance of diamond back moth to abamectin and field control experiments in Yunnan. Journal of Huazhong Agricultural University, v.20, p.426-430, 2001.

Recebido em 29 de maio de 2006 e aprovado em 14 de fevereiro de 2007 